\section{Density of Frequencies in Lattice Dynamics}

THE preceding communication by Dr. Nagendra Nath is one of a series attempting to defend Raman's interpretation of the diffuse spots in X-ray photographs of crystals by discrediting the thermal theory accepted by other investigators of this phenomenon. Raman insists that not only is this application of lattice dynamics to X-ray scattering wrong, but also the foundations of lattice dynamies itself. He questions in particular the method of the 'cyclic boundary conditions', which consists in the following: The vibrations of a finite piece of the crystal depend in principle on the shape of the surface and the dynamical conditions prevailing on it. But if the dimensions of the piece are large compared with the single cell, it is plausible that the dependence on the boundary conditions will be inessential, so that these can be replaced by simpler ones (periodicity). The same assumption is made in the theory of radiant heat (black body radiation) and of the electrons in metals. It has never been doubted in these applications, and whoever rejects it would have to reconstruct these extended theories without this simplification.

In order to disprove Raman's objections directly, I have published the solution of a simple example, the finite diatomic chain (linear lattice), and shown that the quantity essential for the thermal applications, the density of the frequencies, is asymptotically the same for the rigorous and the cyclic solution. Dr. D. E. Rutherford, of the University of St. Andrews, who has checked my results by a different and ingenious method, has found that one of the frequencies given in my paper is incorrect; but this is, of course, quite insignificant for the problem considered, as a linear lattice of $1 \mathrm{~cm}$. length has about $10^{8}$ frequencies. In the preceding communication Dr. Nagendra Nath reports that he has worked out (but apparently not published) the same example and found the same mistake (he claims to have found another one, but this statement of his is incorrect). He has also confirmed the splitting of the frequencies into two branches; whether he has found the asymptotic density law is not evident from his letter, as he speaks only about the 'range of frequency', which is not relevant in this connexion.

Dr. Nagendra Nath's subsequent considerations are incomprehensible to me, as for example : "In the exact solution, infinitesimal displacements in not negligible regions have te form as nodal bridges", from which he infers that one has to consider 'real' instead of 'perfect' crystals. For this purpose he formulates four "working hypotheses" the meaning of which is hard to understand (even if a "monochromatic one [spectrum] with a small number of frequencies" is allowed for as a lapsus linguæ). There seems to be no more justification for these hypotheses in the extensive literature on 'real' crystals than in lattice dynamics.

To end this discussion about the cyclic condition, I have asked the help of a mathematical expert, Dr. W. Ledermann, of the University of St. Andrews. $\mathrm{He}$ has proved (see the following letter) a theorem which suffices for the foundation of all purely thermal applications of lattice dynamics (specific heat, thermal expansion, etc.) and in addition shows that a break in the regularity (missing or wrong atoms) at a number of places small compared with the total number of lattice points does not materially influence the thermodynamical results. This theorem may not be quite sufficient for all problems of crystal theory.
The scattering of $\mathrm{X}$-rays, however, which is the main object of Raman's attack, is quite independent of it. In a paper which will soon appear in Proc. Roy. Soc. I have shown that the formula for the diffuse thermal scattering can be derived in such a way that no other mathematics is used than in the Laue-Bragg theory of ordinary scattering, namely, the fact that certain lattice sums, which are simple generalizations of the Laue function $\sum_{n_{1} n_{2} n_{3}} \exp i\left(n_{1} x_{1}+n_{2} x_{2}+n_{3} x_{3}\right)$, are approximately independent of the boundary for large erystals.

\section{Department of Mathematical Physics, University, Edinburgh.} Max BorN.

IN connexion with the asymptotic distribution of the eigen-frequencies of a cyclic and a finite lattice, the following mathematical theorem will be found helpful :

Let $A$ be a real symmetric matrix of order $n$ in which we display a 'core' $C$ bordered by a single row and column, thus :

$$
A=\left[\begin{array}{ll}
C & h \\
h^{\prime} & \eta
\end{array}\right]
$$

where $C$ is a symmetric matrix of order $n-1, h$ a column, $h^{\prime}$ its transpose and $\eta$ a number. The latent roots of $A$ are $n$ real numbers $\mu_{1}, \mu_{2}, \ldots ., \mu_{n}$. Let the number of latent roots which lie in the interval $\alpha \leqslant \mu \leqslant \beta$ be denoted by $P(\alpha, \beta)$. We wish to compare the latent roots of $A$ with those of the matrix

$$
B=\left[\begin{array}{ll}
C & k \\
k^{\prime} & x
\end{array}\right],
$$

which differs from $A$ only in the last row and column. If the number of latent roots of $B$ in the same interval be denoted by $Q(\alpha, \beta)$, it can be shown that

$$
|P(\alpha, \beta)-Q(\alpha, \beta)| \leqslant 2 \text {. }
$$

This inequality is independent of the particular border $(h, \eta)$ or $(k, x)$ and is independent of $n$. The result is especially interesting for large values of $n$, as it provides a proof of the plausible fact that the distribution of the latent roots of a large symmetric matrix is not materially changed by the modification of the last row and column. When the last $p$ rows and columns are modified, the inequality reads

$$
|P(\alpha, \beta)-Q(\alpha, \beta)| \leqslant 2 p \text {. }
$$

Hence if $p$ be small compared with $P$ or $Q$, it follows that $P$ and $Q$ are asymptotically equal.

The bearing of this theorem on the problem of crystal vibration is as follows : the $N$ atoms of the lattice are divided into internal atoms and boundary atoms, according as the atom has a complete set of acting neighbours or not. Instead of numbering the atoms with three indices corresponding to their spatial arrangement (as is usual in lattice theory) we imagine the atoms to be enumerated in a simple sequence, starting somewhere in the interior and taking first all the internal atoms and then adding the boundary atoms. As the vibration is vectorial, there are three equations of motion for each particle. The number, $p$ say, of 'boundary' equations is of smaller order of magnitude than that of the 'internal' equations $\left(p \sim N^{2 / 3}\right)$. The matrix whose latent roots are the squares of the frequencies is of the type $A$ considered above, involving a border of $p$ rows and columns. The introduction of the cyclic condition causes a change only in the border elements of the matrix, and the theorem regarding the asymptotic 